\title{
Distributed Automatic Generation Control Using Flatness-Based Approach for High Penetration of Wind Generation
}

\author{
Maryam Hassani Variani, Student Member, IEEE, and Kevin Tomsovic, Fellow, IEEE
}

\begin{abstract}
To allow for high penetration of distributed generation and alternative energy units, it is critical to minimize the complexity of generator controls and the need for close coordination across regions. We propose that existing controls be replaced by a two-tier structure of local control operating within a global context of situational awareness. Flatness as an extension of controllability for nonlinear systems is a key to enable planning and optimization at various levels of the grid in this structure. In this study, flatness-based control for automatic generation control (AGC) of a multi-machine system with high penetration of wind energy is investigated. The local control tracks the reference phase which is obtained through economic dispatch at the global control level. As a result of applying the flatness-based method, the $\boldsymbol{n}$ machine system is decoupled into $n$ linear controllable systems in canonical form. Therefore, the control strategy results in a distributed AGC formulation which is significantly easier to design and implement compared to conventional AGC. Practical constraints such as generator ramping rates can be considered in designing the local controllers. The proposed strategy demonstrates promising performance in mitigating frequency deviations and the overall structure could facilitate operation of other nontraditional generators.
\end{abstract}

Index Terms-Automatic generation control (AGC), flatness, frequency regulation, trajectory generation, trajectory tracking, wind power.

\section{NOMENCLATURE}

$\delta_{i} \quad$ Rotor electrical angle, in rad (subscript $i$ denotes the $i$ th generator).

$\omega_{i} \quad$ Rotational speed of rotor, in $\mathrm{rad} / \mathrm{s}$.

$\omega_{s} \quad$ Machine electrical synchronous speed, in $\mathrm{rad} / \mathrm{s}$.

$P_{g v i} \quad$ Governor power, in p.u.

$P_{m i} \quad$ Mechanical power, in p.u.

$P_{i}^{r e f} \quad$ Speed changer position.

$E_{i} \quad$ Voltage behind reactance, in p.u.

Manuscript received June 28, 2012; revised November 23, 2012 and January 31, 2013; accepted March 10, 2013. Date of publication April 26, 2013; date of current version July 18,2013. This work was supported in part by GCEP at Stanford University and in part by the Engineering Research Center Program of the National Science Foundation and the Department of Energy under NSF Award Number EEC-1041877 and the CURENT Industry Partnership Program. Paper no. TPWRS-00738-2012.

The authors are with the Min H. Kao Department of Electrical Engineering and Computer Science, The University of Tennessee, Knoxville, TN 37996 USA (e-mail: mhassani@utk.edu; tomsovic@eecs.utk.edu).

Color versions of one or more of the figures in this paper are available online at http://ieeexplore.ieee.org.

Digital Object Identifier 10.1109/TPWRS.2013.2257882

\author{
$V_{i} \angle \theta_{i} \quad$ Machine terminal voltage, in p.u. and rad. \\ $x_{d i}^{\prime} \quad$ Direct axis transient reactance, in p.u. \\ $H_{i} \quad$ Inertia constant, in seconds. \\ $D_{i} \quad$ Damping constant, in p.u. \\ $R_{i} \quad$ Slope of the machine speed-droop characteristic, \\ in p.u. \\ $\tau_{g i}, \tau_{T i} \quad$ Governor and turbine time constants in seconds. \\ $B_{f} \quad$ Frequency bias setting, in MW/0.1 Hz.
}

\section{INTRODUCTION}

$\mathbf{T}$ HE rapid introduction of wind power has begun to impact overall power system control, and particularly frequency control. As a fundamental characteristic of electric power operations, frequency of the system deviates from its nominal value due to generation-demand imbalance. Conventional generators, in which the turbine rotational speed is nearly constant, provide inertia and governor response against frequency deviations; however, the speed of a wind turbine is not synchronous with the grid and is usually controlled to maximize active power production. Therefore, wind plant power production is not inherently coupled to the system frequency, and historically, wind plants have not been required to participate in frequency regulation. Still, modern wind plants offer limited ability to contribute in frequency regulation within few seconds after loss of generation [1]. With increased penetration of wind energy, system operators have begun to study the performance of the primary frequency response. The California ISO frequency response study shows that the reduced system inertia due to penetration of wind units has an impact on the initial rate of change of frequency but it has little impact on the severity of the frequency excursion and settling frequency. Inertia controls from wind generation can significantly improve the frequency nadir but they do relatively little to correct a shortage in the amount of available response. Unlike inertial response, wind plant governor like control will significantly improve frequency nadir and settling frequency. This control requires the wind plants to work below available power [2]. According to the investigation of wind generation penetration in the ERCOT market, the percentage increase in regulation requirements has been found to be equal to the percentage wind penetration on a capacity basis. The regulation needs increase much more for certain times of the year [3]. Another assessment of frequency 
control considered changing a fraction of the online turbine capacity that provides primary and secondary control. This study showed that adequate reserve to cover expected variations of wind power is not sufficient on its own. In fact, proper dynamic characteristics and control capabilities are as important as the level of reserves [4].

The amount of secondary control response capability required and the rate at which it must be delivered have historically been functions of the daily load forecast, allowance for error in the forecast, and provision for contingencies. The introduction of variable resources clearly adds a new and potentially large component to the requirement for secondary response with respect to both amount and rate of delivery. The correct operation of the system for load frequency control (LFC) to handle as much as possible of the imperfection results in minimizing the use of primary response capability [4]. If secondary control is exhausted due to wind, solar or load variability, the actions of primary frequency control will reduce primary frequency response capability for responding to the faults [2]. Secondary control action is based on the assumption that frequency error throughout a balancing authority is identical. This assumption may not be well suited for systems with high wind penetration because larger imbalances may occur at locations with high installed wind capacity [5]. On the other hand, improvements in wide area measurements allow for more distributed secondary control. Thus, a high proportion of wind powered generation will require renewed attention to secondary control capability.

AGC, secondary frequency control, has been conventionally performed by integrating the area control error (ACE), which acts on the load reference settings of the governors. The integral of square error (ISE) is used in [6] to find the optimum gain for the controller. Performance of other classical control methods such as proportional-integral (PI), integral-derivative (ID), proportional-integral-derivative (PID) and integral-double derivative (IDD) controllers is investigated in [7]. All these approaches are adequate for the traditional centralized system controls but suffer from the need for careful coordination of local controllers with overall system objectives.

In the present study, a flatness-based approach is applied to multi-machine AGC. The performance of the proposed control system is investigated in presence of penetration of wind generation. Flat systems were first introduced by Fliess [8] using the formalism of differential algebra. In differential algebra, a system is viewed as a differential field generated by a set of variables. The system is said to be flat if one can find a set of variables, called the flat outputs, such that the system is algebraic over the differential field generated by the set of flat outputs. The flatness-based approach is well adopted to control systems in two levels of planning, trajectory generation, and tracking the desired trajectories. In the proposed structure, the $n$-machine system is split into $n$ linear controllable systems. Consequently, the control strategy is significantly easier to implement relative to conventional AGC. The flatness property of synchronous machines is introduced in [9] and applied to a single machine connected to infinite bus. The approach is extended in this work to establish a two-tier structure in a multi-machine system to control the frequency and tie-line power flow considering the overall system reliability, speed, and robustness. In local control, individual components and individual loads operate in a manner to follow some desired trajectory based on local observations. The global control, on the other hand, refers to the desired trajectory which is determined by the context of the overall system needs.

\section{Flat SySTEMS}

When a system is flat, it is an indication that the nonlinear structure of the system is well characterized and one can exploit that structure in designing control algorithms for motion planning, trajectory generation, and stabilization. One major property of differential flatness is that the state and input variables can be directly expressed in terms of the flat output and a finite number of its derivatives. In other words, a system is flat if we can find a set of flat outputs (equal in number to the number of inputs) such that all states and inputs can be determined from these outputs without integration [10].

Consider

$$
\dot{x}=f(x, u) \quad\left(x \in R^{n}, u \in R^{m}\right) .
$$

The dynamic feedback linearizability of (1) means the existence of a regular dynamic compensator

$$
\begin{aligned}
& \dot{z}=a(x, z, v) \\
& u=b(x, z, v) \quad\left(z \in R^{q}, v \in R^{m}\right)
\end{aligned}
$$

and a diffeomorphism

$$
\xi=\Xi(x, z) \quad\left(\xi \in R^{n+q}\right)
$$

such that (1) and (2), whose $(n+q)$ dimensional dynamics is given by

$$
\begin{aligned}
\dot{x} & =f(x, b(x, z, v)) \\
\dot{z} & =a(x, z, v)
\end{aligned}
$$

becomes, according to (3), a constant linear controllable system $\dot{\xi}=F \xi+G v$.

Up to a static state feedback and a linear invertible change of coordinates, this linear system may be written in Brunovsky canonical form

$$
\begin{gathered}
y_{1}^{\left(\alpha_{1}\right)}=v_{1} \\
\cdot \\
\cdot \\
y_{m}^{\left(\alpha_{m}\right)}=v_{m}
\end{gathered}
$$

Then it can be deduced that

$$
\begin{aligned}
x & =A\left(y, \dot{y}, \ldots, y^{(\alpha-1)}\right) \\
u & =B\left(y, \dot{y}, \ldots, y^{(\alpha)}\right)
\end{aligned}
$$

where $\left(y, \dot{y}, \ldots, y^{(\alpha-1)}\right)$ corresponds symbolically to $\left(y_{1}, \ldots, y_{1}^{\left(\alpha_{1}-1\right)}, \ldots, y_{m}, \ldots, y_{m}^{\left(\alpha_{m}-1\right)}\right)$ and the same for $\left(y, \dot{y}, \ldots, y^{(\alpha)}\right)[8],[11]$.

The dynamic feedback (2) is said to be endogenous if and only if, the converse holds, i.e., if and only if, any component 
of $y$ can be expressed as a real-analytic function of $x$ and $u$ and a finite number of its derivatives

$$
y=C\left(x, u, \dot{u}, \ldots, u^{(\gamma)}\right)
$$

A dynamics (1) which is linearizable via such an endogenous feedback is said to be (differentially) flat; $y$ is called a linearizing or flat output.

The flatness property may be very useful when dealing with trajectories: from the $y$ trajectories, $x$ and $u$ trajectories are immediately deduced. These properties permit a straightforward open loop path tracking. The equivalence of the flat system with a controllable linear system via an endogenous feedback yields a feedback stabilization of the desired trajectory. Precisely, according to the flat output properties, system trajectories joining a collection of points with given velocities, acceleration, jerks, etc., are easily generated. This replaces difficult dynamical computations by statistical interpolation techniques [12].

\section{A. Trajectory Generation}

Due to flat outputs properties, all trajectories $(x(t), u(t))$ satisfying the system differential equation can be interpreted in terms of the flat output and its derivatives (6). Considering the problem of steering from an initial state to a final state, the components of the flat output $y_{i}, i=1, \ldots m$ are parameterized by

$$
y_{i}(t):=\sum_{j} A_{i j} \lambda_{j}(t)
$$

where $\lambda_{j}(t), j=1, \ldots, \alpha$ are basic functions. Thus, the problem of steering from an initial state $x\left(t_{i}\right)$ to $x\left(t_{f}\right)$ reduces to find the coefficient $A_{i j}$ in the following system of equations:

$$
\begin{array}{ccc}
y_{i}\left(t_{0}\right) & = & \sum_{j} A_{i j} \lambda_{j}\left(t_{0}\right) \\
\cdot & \cdot \\
y_{i}^{\left(\alpha_{i}-1\right)}\left(t_{0}\right)= & \sum_{j} A_{i j} \lambda_{j}^{\left(\alpha_{i}-1\right)}\left(t_{0}\right) \\
y_{i}\left(t_{f}\right) & = & \sum_{j} A_{i j} \lambda_{j}\left(t_{f}\right) \\
\cdot & \cdot \\
y_{i}^{\left(\alpha_{i}-1\right)}\left(t_{f}\right) & = & \sum_{j} A_{i j} \lambda_{j}^{\left(\alpha_{i}-1\right)}\left(t_{f}\right) .
\end{array}
$$

It is observed that the problem reduces to solving simple algebraic equations. The multidimensional case follows by repeatedly applying the one-dimensional case, since the algorithm is decoupled in the component of the flat output [10], [13].

\section{B. Trajectory Tracking}

In a flat system, tracking the desired trajectory is ensured using the fact that the flat system is equivalent to a trivial system $y^{(\alpha)}=v$ by endogenous dynamic feedback. By setting $\left(y^{*}\right)^{(\alpha)}=v^{*}$, it suffices then to set

$$
v=v^{*}-\sum_{j=1}^{\alpha-1} K_{j} e^{(j)}
$$

with appropriate gain matrix $K=\left[K_{1}, \ldots, K_{\alpha-1}\right]$ and $e=y-$ $y^{*}$ the error term in this equation. Linear control methods such as pole-placement and linear quadratic regulator (LQR) can be applied to find the $K$ coefficients. Similar to trajectory generation for multidimensional case, trajectory tracking follows by repeatedly applying the one-dimensional case [10], [13].

\section{FREQUENCY REGULATION}

System frequency deviates from the nominal setting whenever there is imbalance between generation and load. The imbalance will be drawn from the kinetic energy stored in the rotating masses of the generators. Local governor control is the primary control loop to ensure balance. In order to maintain system frequency at the nominal value and schedules between control areas, a secondary control loop, AGC, coordinates the unit raise and lower signals.

\section{A. Conventional AGC}

The supplemental control serves several functions, including: restoration of the nominal frequency, maintenance of the scheduled interchanges between authority areas and provision for the economic dispatch of units. The coordination among areas is achieved by defining the so called ACE. A frequency bias setting, $10 B_{f}$, is multiplied by the frequency deviation, $\Delta f$, which is subtracted from the deviation of tie flows to obtain ACE. The ACE is integrated over time and this signal is used to determine the generator set points. The control center gathers the relevant frequency and power flow information, calculates the $\mathrm{ACE}$ and sends the appropriate set point adjustments for each of the units on AGC. Therefore, set point controls are discrete. In North America, the AGC signals are fed to the units typically, about once every 2-4 s [14]. Economic dispatch is performed once every 5 min to calculate plant based points and participation factors for plants on secondary control. Conventionally, each control area of an interconnected system is controlled in a similar manner, but independently of the other control areas. That is, the control of generation in the interconnected system is "area-wise decentralized" [15].

\section{B. Governor-Turbine-Generator Model}

Considering the governor-prime-mover-rotating mass/load model block diagram in [16], a multi-machine system to analyze AGC performance can be described by a fourth-order model in (11)-(14). Since the focus of this study is only on frequency response, it is appropriate to assume that the voltage regulator and other dynamics within the machine are fast compared to the phenomena of interest [17]:

$$
\begin{aligned}
\dot{\delta}_{i} & =\omega_{i}-\omega_{s} \\
\dot{\omega}_{i} & =\frac{1}{2 H_{i}}\left[\begin{array}{c}
P_{m i}-\frac{E_{i} V_{i}}{x_{d i}^{\prime}} \sin \left(\delta_{i}-\theta_{i}\right) \\
-D_{i}\left(\omega_{i}-\omega_{s}\right)
\end{array}\right] \\
\dot{P}_{g v i} & =\frac{1}{\tau_{g_{i}}}\left(P_{i}^{r e f}-\frac{\omega_{i}-\omega_{s}}{R_{i} \omega_{s}}-P_{g v i}\right) \\
\dot{P}_{m i} & =\frac{1}{\tau_{T_{i}}}\left(P_{g v i}-P_{m i}\right) .
\end{aligned}
$$

In this model, the active power output at the generator internal nodes is stated as a function of terminal voltage, voltage behind 
the reactance and $x_{d}^{\prime}[18]$. Terminal voltage magnitude and angle depend on the network equations. Since the flux decay dynamics are neglected, terminal voltage can be calculated using algebraic equations.

Speed changer position, $P_{i}^{r e f}$, is conventionally calculated through integration of ACE. The main challenge in this method is the design of the integral controller and the coordination across areas. This becomes more challenging in the presence of wind farms in the system as the energy generated by these units varies rapidly, which may result in misleading ACE signals.

\section{Flatness-BASED AGC}

Based on (11)-(14) and considering $\delta=\left[\delta_{1}, \ldots, \delta_{i}, \ldots, \delta_{n}\right]^{\prime}$ as the flat output set, the flat outputs and their derivative up to degree four for a multi-machine system can be derived as follows:

$$
\begin{gathered}
\dot{\delta}_{i}=\omega_{i}-\omega_{0} \\
\ddot{\delta}_{i}=\frac{1}{2 H_{i}}\left[\begin{array}{c}
P_{m i}-\frac{E_{i} V_{i}}{x_{d i}^{\prime}} \sin \left(\delta_{i}-\theta_{i}\right) \\
-D_{i}\left(\omega_{i}-\omega_{s}\right)
\end{array}\right] \\
\delta_{i}^{(3)}=\frac{1}{2 H_{i}}\left[\begin{array}{c}
\frac{1}{\tau_{T i}}\left(P_{g v i}-P_{m i}\right)-D_{i} \ddot{\delta} \\
-\frac{E_{i} V_{i}}{x_{d i}^{\prime}} \dot{\delta}_{i} \cos \left(\delta_{i}-\theta_{i}\right)
\end{array}\right] \\
\delta_{i}^{(4)}=\frac{1}{2 H_{i}}\left[\begin{array}{c}
\frac{1}{\tau_{T i} \tau_{g i}}\left(P_{i}^{r e f}-\frac{1}{R \omega_{s}}-P_{g v}\right) \\
-\frac{1}{\tau_{T i}^{2}}\left(P_{g v i}-P_{m i}\right) \\
-\frac{E_{i} V_{i}}{x_{d i}^{\prime}}\left(\ddot{\delta}_{i} \cos \left(\delta_{i}-\theta_{i}\right)\right. \\
\left.-\left(\dot{\delta}_{i}\right)^{2} \sin \left(\delta_{i}-\theta_{i}\right)\right)-D_{i} \delta_{i}^{(3)}
\end{array}\right] .
\end{gathered}
$$

The algebraic relations between the state variables, input, flat outputs and their derivatives, verify that $\delta$ is the flat output in this system as stated in (15)-(18). The algebraic functions $A, B$ and $C$ can be defined as follows:

$$
\begin{aligned}
& x_{i}=A\left(\delta_{i}, \dot{\delta}_{i}, \ldots, \delta_{i}^{(3)}\right) \\
& u_{i}=B\left(\delta_{i}, \dot{\delta}_{i}, \ldots, \delta_{i}^{(4)}\right) \\
& \delta_{i}=C\left(x_{i}, u_{i}\right)
\end{aligned}
$$

where $x_{i}=\left[\delta_{i}, \omega_{i}, P_{g v i}, P_{m i}\right]^{\prime}$ and $u_{i}=P_{i}^{r e f}$. Therefore, the compensator for the system in each area can be defined as

$$
\begin{aligned}
& \dot{z}_{i 1}=z_{i 2} \\
& \dot{z}_{i 2}=z_{i 3} \\
& \dot{z}_{i 3}=z_{i 4} \\
& \dot{z}_{i 4}=v_{i}
\end{aligned}
$$

where $z_{i 1}=\delta_{i}$ and $\delta_{i}^{(4)}=v_{i}$. According to (21), one sees that the dynamics of a multi-machine system can be split into $n$ linear controllable subsystems. The trajectory generation and the asymptotic tracking of the desired trajectory, rotor angle, is studied in the following to find the control input, $v_{i}$, for each subsystem.

\section{A. Trajectory Generation for Multi-Machine AGC System}

An important role of AGC is to allocate generation so that each power source is loaded most economically [15]. In this study, economic dispatch is performed to find the desired operating points. Note that this can be replaced by other methods depending on the system's overall needs. The desired operating point has to be updated at frequent intervals in order to follow load changes and wind generation variations. Here, intervals of $5 \mathrm{~min}$ are considered for economic dispatch as it is used in conventional AGC. The reference points and participation factors are sent to generators every 5 minutes and a smooth trajectory is then planned locally. The planning method described in Section II-A is deployed to generate the optimum path to be followed by trajectory tracking control. Within the five minute intervals, the reference values for rotor angle are updated such that each generator contributes in frequency regulation based on its participation factor. The desired operating point determines the participation factors.

Although the operating points in the system are updated every 5 min, significant wind power changes may occur in the interval and cause the system to deviate from the desired trajectory. In this situation, the trajectory can be updated locally by solving simple algebraic equations to keep the system at the new desired trajectory. The trajectory can be updated regularly, e.g., approximately every minute or whenever the deviation from trajectory is larger than a specific value.

\section{B. Trajectory Tracking for Multi-Machine AGC System}

The gain matrix, $K$, in (10) should be designed to restore the nominal frequency and track the scheduled net interchange with desired performance characteristics. Individual units tracking the desired trajectory generated in Section IV-A will guarantee overall system performance. The trivial system of (21) for each area is the key to achieve tracking of the desired trajectory. In general any simple linear control method can be applied to find the gain. In this work, the LQR method is employed which allows consideration of practical constraints related to AGC [19].

The obtained $K$ leads to asymptotic tracking of the desired trajectory. The practical constraints considered in this study are:

- Generator ramping rate constraint (GRC) which limits the rate of generation increase/decrease. In LQR method, this constraint can be considered by choosing a large value for $R$, which is representative of cost of the control.

- Raise/lower signals are sent to the governor every 2 s. Therefore, continuous optimal control may not be optimal for the system in practice. In order to design the digital control law for this continuous time systems, the flat system is discretized using 2-s samples. The discretization process assumes that the control input $v(t)$ to the continuous plant is switched only at times $2 k$ and it is held constant between switchings [19]. In North America, AGC is typically executed once every 2 to $4 \mathrm{~s}$. However, the control action in flatness approach is based on local measurements and due to a large sampling rate of modern units, measurement does not restrict the frequency of sending controller signal. In the other words, the control signal can be sent to governor as frequent as the governor limits allow.

\section{Summary of Approach}

Fig. 1 shows the schematic control diagram of the proposed approach. Desired operating points are determined at the global level control using the economic dispatch or other methods, while the trajectory is generated at the local control level. Rotor angle and frequency are the quantities requiring monitoring in 


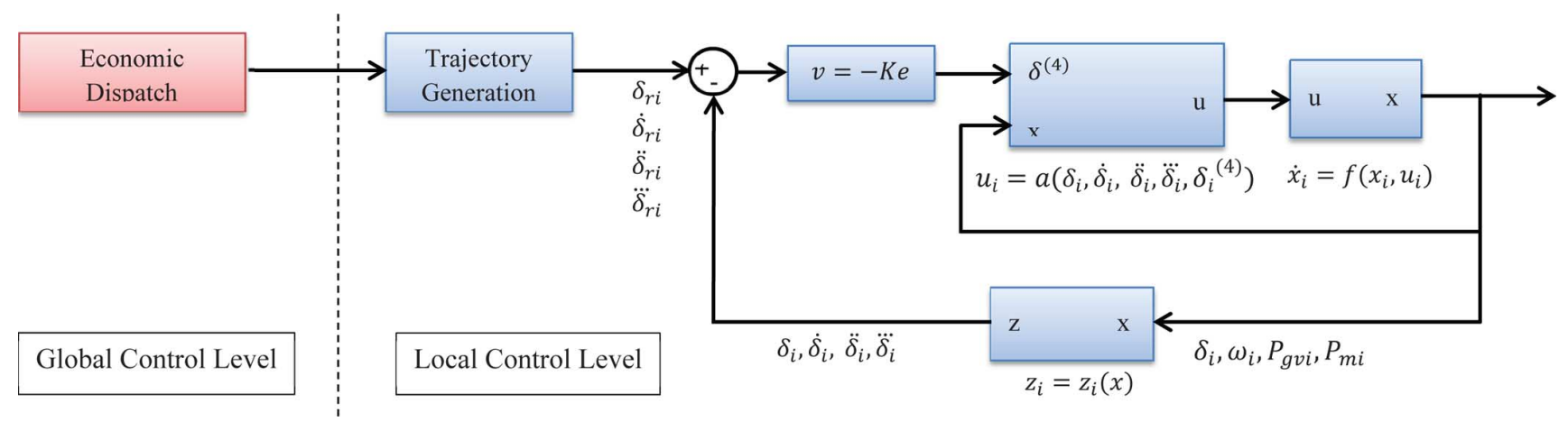

Fig. 1. Flatness-based control block diagram.

this scheme. Due to the lack of a direct measurement of rotor angle, this parameter is assumed estimated using the measurements of a phasor measurement unit (PMU) [20]. In this study, rotor angle is estimated based on the terminal voltage angle and generator active and reactive powers as shown in (22) and (23):

$$
\begin{aligned}
& I_{i}=I_{D i}+j I_{Q i}=\frac{\left(P_{G i}-j Q_{G i}\right)}{V_{i} e^{-j \theta_{i}}} \\
& \delta_{i}=\angle\left(V_{i} e^{j \theta_{i}}+j x_{d i}^{\prime}\left(I_{D i}+j I_{Q i}\right)\right) .
\end{aligned}
$$

The measured and estimated quantities are compared with the reference values and the control signal is generated through a trajectory tracking approach at the generator level. In summary, the planning is performed at the global level and the trajectory is generated and tracked locally using closed loop control.

\section{Simulation Results}

In this section, the proposed flatness-based AGC is evaluated on a 3-area, 10-machine and the 39-bus system shown in Fig. 2 and is compared with conventional AGC [21]. The total load in this system is assumed to be $5.483 \mathrm{GW}$ and economic dispatch is performed to find the scheduled active power generation. In order to evaluate the performance of the controller in presence of penetration of wind energy, the wind power profile shown in Fig. 3 is applied to the test system. The wind power has an average value of $500 \mathrm{MW}$ and the fluctuations are about $\pm 5 \%$ of the average power. Frequency deviation in frequency domain, considering spatial filtering of geographically dispersed wind turbines in a wind farm, is used to generate this wind power profile [22]. Two scenarios are studied:

- Scenario 1: 10\% penetration of wind power generation in area 2.

- Scenario 2: 20\% penetration of wind power generation in areas 1 and 2 .

Based on the Western Wind and Solar Integration Study (WWSIS) results, the addition of every $3 \mathrm{MW}$ of wind generation was accomplished with a $2-\mathrm{MW}$ de-commitment and a 1 -MW reduction in other generation. Therefore for $500-\mathrm{MW}$ additional wind production, the $2 / 3$ de-commitment objective is $333 \mathrm{MW}$ and the $1 / 3$ re-dispatch objective is $167 \mathrm{MW}$. De-commitment of the thermal units reduces the contribution of these units in frequency regulation, while dispatching down

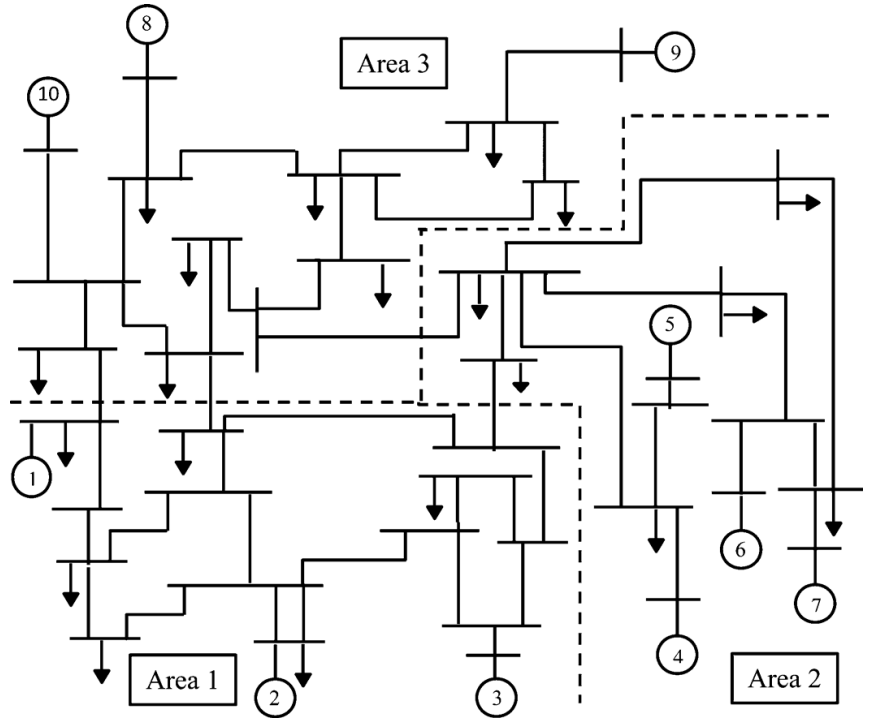

Fig. 2. 10-generator, 39-bus test system.

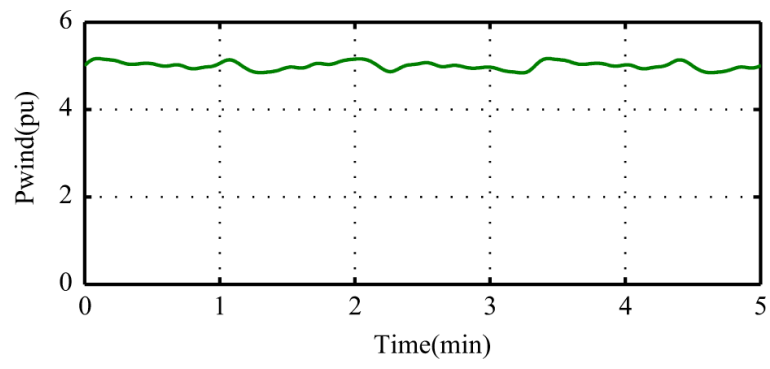

Fig. 3. Wind power (p.u.) - base is 100 MVA.

gives more headroom for secondary control [1]. The original dispatching, the updated dispatching related to scenarios 1 and 2 and generator data are displayed in Table I.

Note in the flatness approach the control areas would not necessarily be the same as today's balancing areas. Smaller balancing areas can be selected to improve the controller performance in presence of large scale wind generation at no additional cost of monitoring. The changes in the planning and trajectory generation are investigated in Section V-C. 
TABLE I

GENERATOR DisPatCH IN P.U. (BASE IS 100 MVA)

\begin{tabular}{|c|c|c|c|c|c|c|}
\hline Area & Gen & $\begin{array}{c}\text { Original } \\
\text { Dispatch }\end{array}$ & $\begin{array}{c}\text { Scenario 1 } \\
\text { Dispatch }\end{array}$ & $\begin{array}{c}\text { Scenario 2 } \\
\text { Dispatch }\end{array}$ & $\begin{array}{c}\text { Inertia } \\
H(\text { sec })\end{array}$ & $\begin{array}{c}x_{d}^{\prime} \\
(\mathrm{pu})\end{array}$ \\
\hline \multirow{3}{*}{1} & 1 & 5.620 & 5.620 & 5.620 & 70.0 & 0.020 \\
\cline { 2 - 7 } & 2 & 3.727 & 3.727 & 2.454 & 30.3 & 0.070 \\
\cline { 2 - 7 } & 3 & 3.727 & 3.727 & 0 & 35.8 & 0.053 \\
\hline \multirow{4}{*}{2} & 4 & 7.481 & 7.481 & 7.481 & 28.6 & 0.044 \\
\cline { 2 - 7 } & 5 & 7.796 & 7.796 & 7.796 & 26.0 & 0.132 \\
\cline { 2 - 7 } & 6 & 5.796 & 5.092 & 5.092 & 34.8 & 0.050 \\
\cline { 2 - 7 } & 7 & 4.296 & 0 & 0 & 26.4 & 0.049 \\
\hline \multirow{3}{*}{3} & 8 & 4.296 & 4.296 & 4.296 & 24.3 & 0.057 \\
\cline { 2 - 7 } & 9 & 4.296 & 4.296 & 4.296 & 34.5 & 0.057 \\
\cline { 2 - 7 } & 10 & 7.797 & 7.797 & 7.797 & 20.0 & 0.044 \\
\hline
\end{tabular}
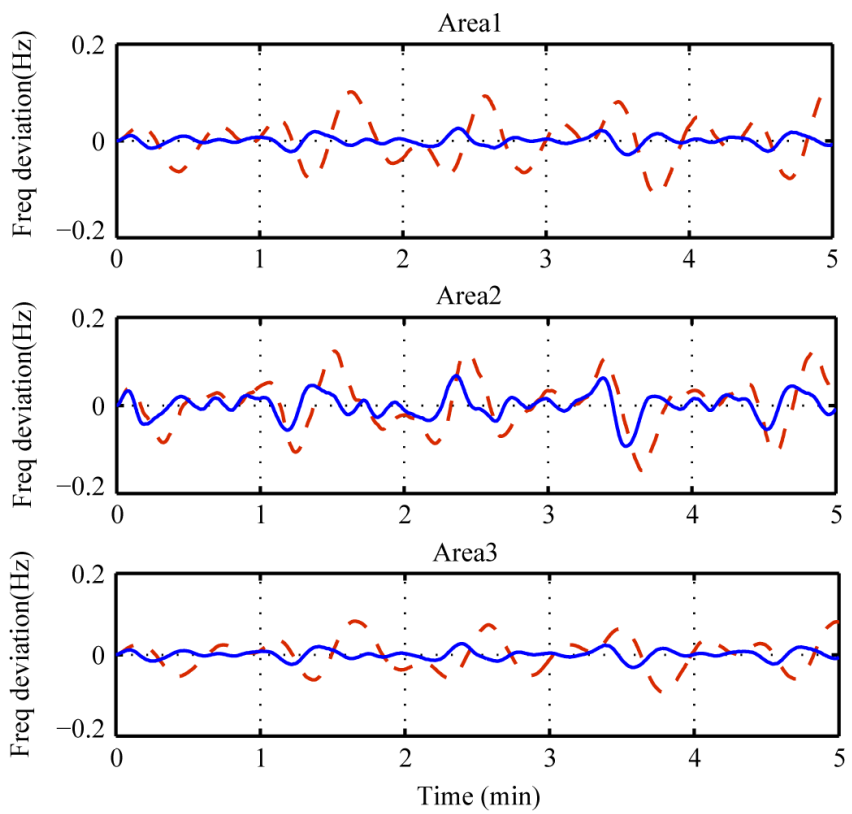

Fig. 4. Frequency deviation with conventional (dash line) and flattness_based (solid line) with $10 \%$ penetration.

\section{A. Scenario 1}

In this scenario, the wind power profile is added in area 2, which is about $10 \%$ of the total load. Fig. 4 displays the average frequency deviations in each area. The total mechanical power values in each area are shown in Fig. 5. Tie line flow deviations from the scheduled values are display in Fig. 6. As observed, the flatness approach results in improved performance in mitigating both the frequency and tie flow deviations while the mechanical power changes do not excess the ramping rate limits of the generators. Comparison of frequency deviations and tie flow deviations in three areas shows that, with flatness-based approach the control actions occur primarily in the area where the wind farm is located. In other words, the wind power fluctuations are damped locally. It is worth mentioning that in the flatness-based approach, the average of the frequency in areas is only calculated for clear presentation. As stated in Section IV-C, the frequency is measured locally and compared with a reference value for each generator.
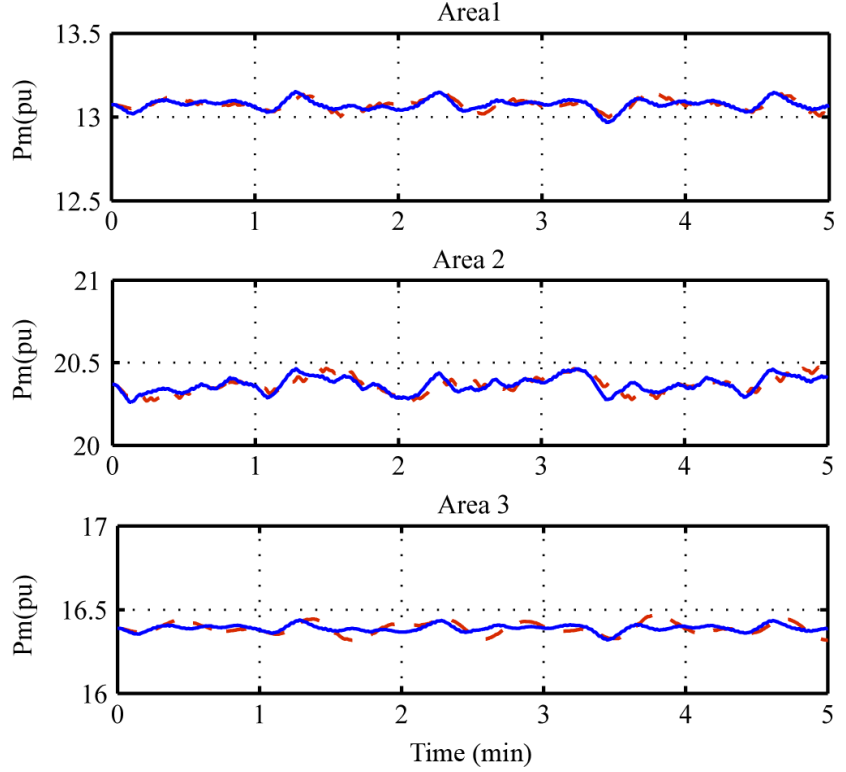

Fig. 5. Mechanical power with conventional (dash line) and flattness_based (solid line) with $10 \%$ penetration.
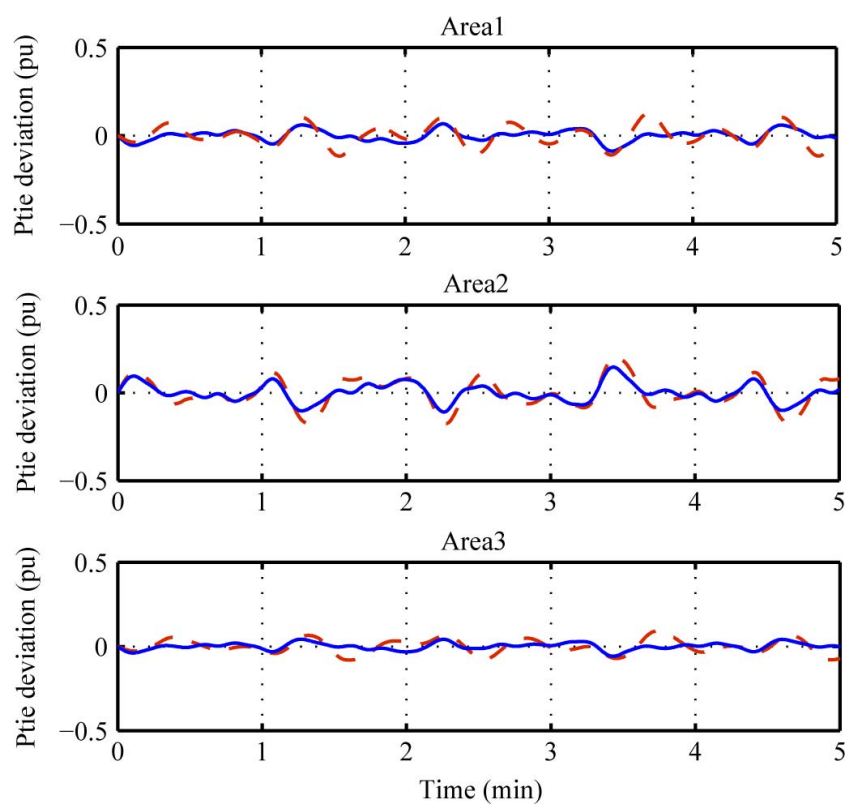

Fig. 6. Tie flow with conventional (dash line) and flattness_based (solid line) with $10 \%$ penetration.

\section{B. Scenario 2}

In this scenario, a wind farm is in both areas 1 and 2 . The average frequency deviations in each area, total mechanical power values in each area and tie flow fluctuations are shown in Figs. 7-9. The first observation is that higher penetration of wind power results in greater frequency and tie flow deviations in the system. Also, comparison of the two scenarios shows that the effectiveness of the flatness-based approach is more noticeable with the higher penetration of wind power. 

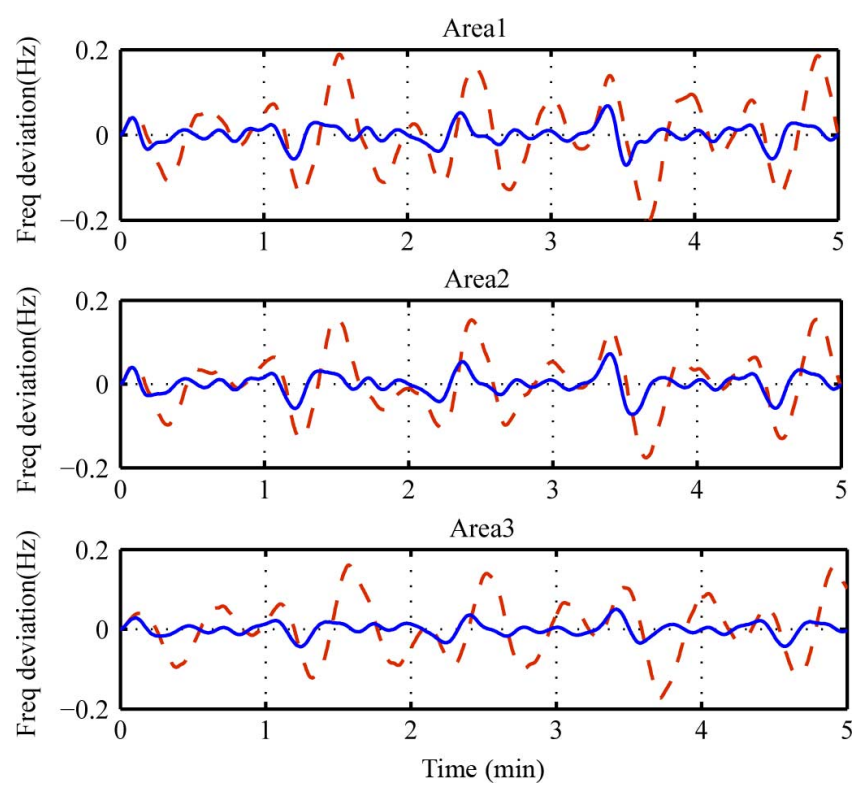

Fig. 7. Frequency deviation with conventional (dash line) and flattness based (solid line) with $20 \%$ penetration.
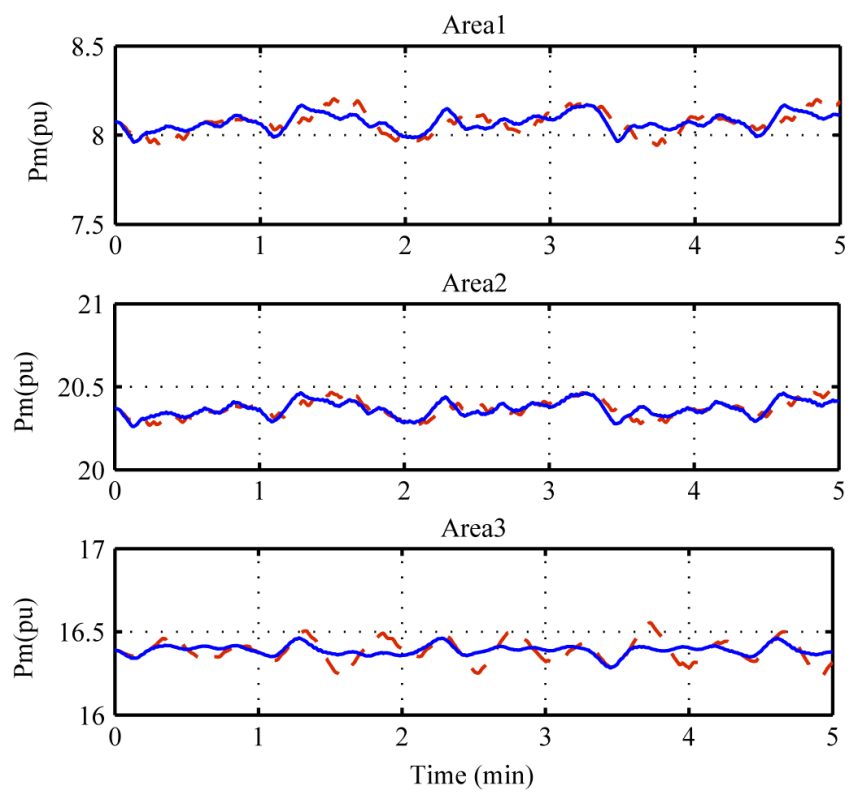

Fig. 8. Mechanical power with conventional (dash line) and flattness_based (solid line) with $20 \%$ penetration.

\section{Planning}

Simulation results shown in Section V-A and B are assumed to be performed in 5-min intervals with a constant desired operating point. In this section, the concept of trajectory generation that reflects the system needs is demonstrated. Two generators in area 2 are re-dispatched so that the scheduled value for generator 5 is decreased by 0.2 p.u. and the scheduled value for generator 7 is increased by the same amount. Fig. 10 shows two different trajectories, one with a step change in the rotor angle trajectory and the other one based on generating a smooth trajectory. The actual rotor angles are also shown in the same figure. Fig. 11 displays the frequency deviations in two generators related to
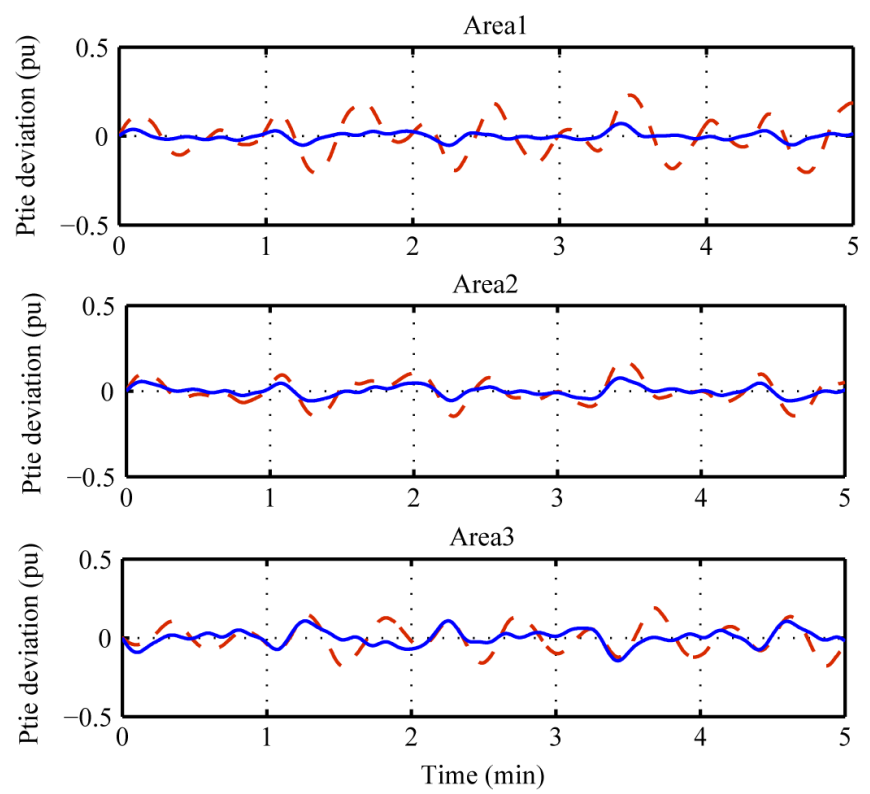

Fig. 9. Tie flow with conventional (dash line) and flattness based (solid line) with $20 \%$ penetration.
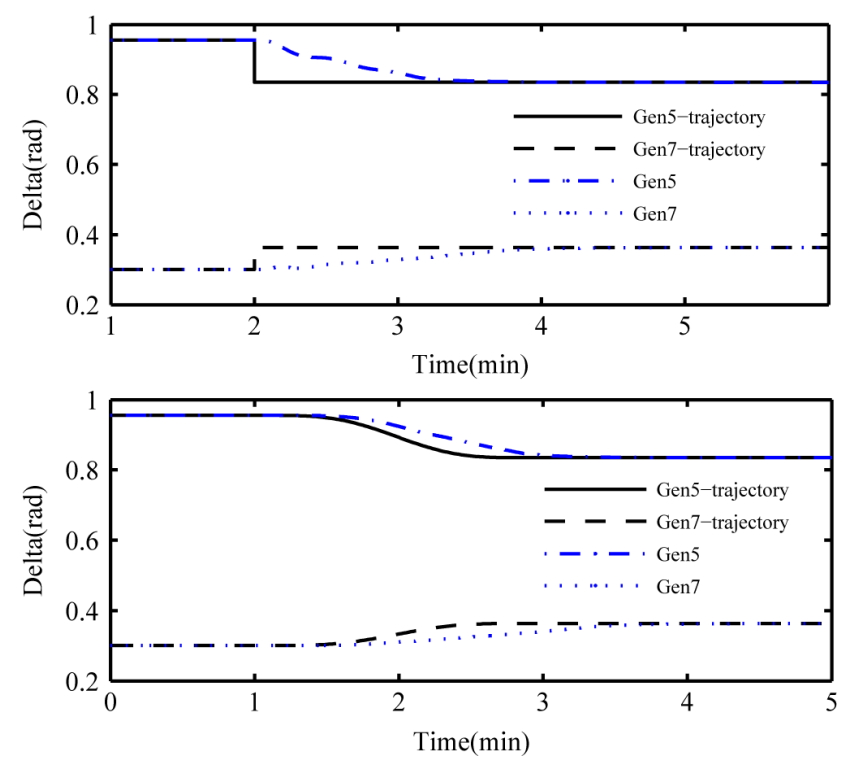

Fig. 10. Rotor angles and trajectories in Area 2.

two trajectories. It is observed that the smooth trajectories result in improved frequency and rotor angle deviations and the controller tracks the desired trajectories well. The lower deviations decrease the control effort required to keep the system at the desired operating point.

\section{CONCLUSIONS}

This paper presents a flatness-based method to control frequency and power flow for multi-area power systems. The two level control consisting of trajectory generation and trajectory tracking replaces conventional AGC. This approach can also replace conventional area based frequency control. As an important feature of the proposed approach, the set of nonlinear equations corresponding to an $n$-machine system is decoupled into $n$ 


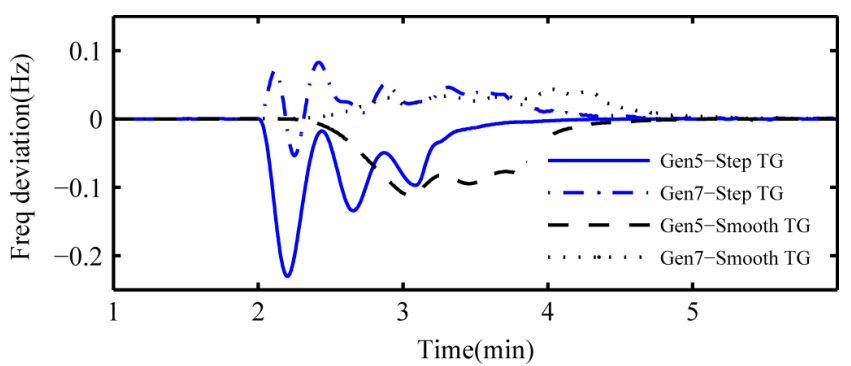

Fig. 11. Frequency deviation in Area 2.

linear controllable sub-systems. Therefore, the proposed AGC is easier to design and implement. Local linear controllers are designed for each sub-system to maintain the frequency at nominal value and to keep power flows near scheduled values. The main requirement is the availability of PMU measurements. The flatness-based control method demonstrates promising performance in mitigating frequency and tie-line flow deviation. This approach also provides a platform for non-conventional units to contribute to load following and frequency control.

\section{REFERENCES}

[1] N. W. Miller, K. Clark, and M. Shao, "Frequency responsive wind plant controls: impacts on grid Performance," in Proc. IEEE Power \& Energy Soc. General Meeting, Detroit, MI, USA, Jul. 2011.

[2] N. W. Miller, M. Shao, and S. Venkataraman, "California ISO (CAISO) frequency response study," in GE Energy, Nov. 2011.

[3] R. A. Walling, L. A. Freeman, and W. P. Lasher, "Regulation requirements with high wind generation penetration in the ERCOT market," in Proc. IEEE PES Power Syst. Conf. Expo., Seattle, WA, USA, Mar. 2009.

[4] J. Undrill, Power and Frequency Control as it Relates to Wind-Powered Generation, LBNL Rep., Dec. 2010.

[5] L. Xie, L. A. F. M. Ferreira, P. M. S. Carvalho, J. Liu, B. H. Krogh, N. Popli, and M. D. Ilic, "Wind integration in power systems: operational challenges and possible solutions," Proc. IEEE, vol. 99, no. 1, pp. 214-232, Jan. 2011.

[6] O. I. Elgerd and C. E. Fosha, "Optimum megawatt-frequency control of multiarea electric energy systems," IEEE Trans. Power App. Syst., vol. PAS-89, no. 4, pp. 556-563, Apr. 1970.

[7] J. Nanda, S. Mishra, P. G. Mishra, and K. V. Sajith, "A novel classical controller for automatic generation control in thermal and hydrothermal systems," in Proc. 2010 Joint Int. Conf. Power Electronics, Drives and Energy Syst. (PEDES) \& 2010 Power India, pp. 1-6.

[8] M. Fliess, J. Levine, Ph. Martin, and P. Rouchon, "Flatness and defect of nonlinear systems: introductory theory and examples," Int. J. Control, vol. 61, no. 6, pp. 1327-1361, 1995.

[9] E. C. Anene, U. O. Aliyu, J. Levine, and G. K. Venayagamoorthy, "Flatness-based feedback linearization of a synchronous machine model with static excitation and fast turbine valving," in Proc. IEEE Power Eng. Soc. General Meeting, Jun. 2007, pp. 1-6.
[10] M. Ph, R. M. Murry, and P. Rouchon, Flat Systems, Equivalence and Trajectory Generation Ecole des Mines de Paris, Tech. Rep., Apr. 2003.

[11] H. Sira-Ramirez and S. K. Agrawal, Differentially Flat Systems. New York, NJ, USA: Marcel Dekker, 2004.

[12] M. Fliess, J. Levine, Ph. Martin, and P. Rouchon, "A lie-backlund approach to equivalence and flatness of nonlinear systems," IEEE Trans. Autom. Control, vol. 44, no. 5, pp. 922-937, May 1999.

[13] J. Levine, Analysis and Control of Nonlinear Systems: A FlatnessBased Approach. Heidelberg, Germany: Springer-Verlag, 2009.

[14] K. Tomsovic and M. Venkatasubramanian, "Power system operation and control," in Electrical Engineering Handbook. New York, NY, USA: Elsevier Academic, 2005, pp. 761-778.

[15] P. Kundur, Power System Stability and Control. New York, NY, USA: McGraw-Hill, 1993.

[16] A. J. Wood and B. F. Wollenburg, Power Generation Operation and Control. New York, NY, USA: Wiley, 1996.

[17] A. D. Dominguez-Garcia, "Models for impact assessment of windbased power generation on frequency control," in Control and Optimization Methods for Electric Smart Grids, A. Chakrabortty and M. Ilic, Eds. Berlin, Germany: Springer-Verlag, 2012, vol. 3.

[18] P. Sauer and A. Pai, Power System Dynamics and Stability. Upper Saddle River, NJ, USA: Prentice Hall, 1998.

[19] F. L. Lewis and V. S. Syrmos, Optimal Control. New York, NY, USA: Wiley, 1995.

[20] B. Singh, N. K. Sharma, A. N. Tiwari, K. S. Verma, and S. N. Singh, "Applications of phasor measurement units (PMUs) in electric power system networks incorporated with facts controllers," Int. J. Eng., Sci., Technol., vol. 3, no. 3, pp. 64-82, 2011.

[21] V. Venkatasubramanian, M. Sherwood, V. Ajjarapu, and B. Leonardi, Real-Time Security Assessment of Angle Stability Using Synchrophasors PSERC, Final Project Rep., May 2010.

[22] C. Luo and B. Ooi, "Frequency deviation of thermal power plants due to wind farms," IEEE Trans. Energy Convers., vol. 21, no. 3, pp. 708-716, Sep. 2006.

Maryam Hassani Variani (S'10) received the B.S. and M.S. degrees from Amirkabir University of Technology, Tehran, Iran, in 2006 and 2009, respectively. She is currently pursuing the Ph.D. degree in electrical engineering at The University of Tennessee, Knoxville, TN, USA.

Her main interest is power system control with high penetration of renewable energy systems .

Kevin Tomsovic (F'07) received the B.S. degree in electrical engineering from Michigan Technological University, Houghton, MI, USA, in 1982 and the M.S. and Ph.D. degrees in electrical engineering from the University of Washington, Seattle, WA, USA, in 1984 and 1987, respectively.

Currently, he is Head and CTI Professor of the Department of Electrical Engineering and Computer Science at University of Tennessee, Knoxville, TN, USA, where he also directs the NSF/DOE sponsored ERC CURENT. He was on the faculty of Washington State University from 1992-2008. He held the Advanced Technology for Electrical Energy Chair at Kumamoto University, Kumamoto, Japan, from 1999 to 2000 and was an NSF program director in the ECS division of the Engineering directorate from 2004 to 2006. 